\title{
From minimal gravity to open intersection theory
}

\author{
Alexander Alexandrov $\odot^{*}$ \\ Center for Geometry and Physics, Institute for Basic Science (IBS), Pohang 37673, Korea \\ and ITEP, Moscow, Russia \\ Hisayoshi Murakiఠ ${ }^{\dagger}$ and Chaiho Rim $\odot^{\ddagger}$ \\ Department of Physics, Sogang University, Seoul 04107, Korea
}

(Received 3 July 2019; published 2 December 2019)

\begin{abstract}
We investigated the relation between the two-dimensional minimal gravity (Lee-Yang series) with boundaries and open intersection theory. It is noted that the minimal gravity with boundaries is defined in terms of boundary cosmological constant $\mu_{B}$ and the open intersection theory in terms of boundary marked point generating parameter $s$. Based on the conjecture that the two different descriptions of the generating functions are related by the Laplace transform, we derive the compact expressions for the generating function of the intersection theory from that of the minimal gravity on a disk and on a cylinder.
\end{abstract}

DOI: $10.1103 /$ PhysRevD.100.126002

\section{INTRODUCTION}

The interplay of the KdV hierarchy between twodimensional quantum gravity and intersection theory (IT) for the moduli spaces of closed Riemann surfaces was proposed in [1]. The conjecture of Witten for the IT was proved in [2] by finding the generating function (GF), also known as free energy, of the one-matrix Airy function. Later, the KdV hierarchy was also checked for twodimensional minimal gravity (MG) of Lee-Yang series by constructing the effective GF on genera up to three in [3] using the one-matrix model polynomials.

Similar interplay is noted for the models on the Riemann surfaces with boundaries [4,5]. The GF of the MG with boundaries (BMG) satisfies the relations, similar to the ones known for the open intersection theory (OIT) [6]. These relations include the boundary version of the KdV hierarchy and the string equation, which encode the generalized Virasoro constraints, the analog of the constraints on the closed Riemann surfaces $[7,8]$.

In this paper, we continue the investigation of the interplay between the two-dimensional gravity and intersection theory on the moduli spaces of Riemann surfaces with boundaries.

\footnotetext{
*alexandrovsash@gmail.com

†hmuraki@sogang.ac.kr

*rimpine@ sogang.ac.kr
}

Published by the American Physical Society under the terms of the Creative Commons Attribution 4.0 International license. Further distribution of this work must maintain attribution to the author(s) and the published article's title, journal citation, and DOI. Funded by SCOAP ${ }^{3}$.
While the GF of IT and that of MG satisfy essentially the same equations, including the $\mathrm{KdV}$ hierarchy and the Virasoro constraints, they have perturbative expansions with drastically different properties. The reason is that they belong to different classes of the solutions with different types of analytical properties, which can be related with each other by different types of analytical continuation. It should be noted that the set of $\mathrm{KdV}$ parameters in two theories plays a different role. On the closed Riemann surfaces, the bulk cosmological constant $\mu$ provides the gravitational scaling dimension (GSD) and plays a major role for MG. In addition, the GF is nonanalytic in $\mu$ and therefore, one cannot turn off $\mu$ in MG. On the other hand, $t_{0}$ in IT provides the scaling dimension (SD), which, in general, has nothing to do with $\mu$. As a result, even though both GF are solutions of the same $\mathrm{KdV}$ hierarchy, they are different.

Similar analysis holds for the open KdV hierarchy. BMG needs the boundary cosmological constant $\mu_{B}$ and its open $\mathrm{KdV}$ hierarchy is given in terms of $\mu_{B}$. On the other hand, OIT and its open KdV are described by the boundary parameter $s$. As a result, the open KdV hierarchy of BMG differs from that of OIT. Nevertheless, the two different KdV hierarchies turn out to be closely related to each other: exponentials of the GF of two theories are formally related through the Laplace transform [5].

This paper investigates further the relation between solutions to the open KdV hierarchy of BMG and OIT. As a result of an explicit comparison of the two representations, we obtain an elegant formula for the GF on a disk. Once one finds the solution on the disk, one may further proceed to finding the GF for higher topologies order by order with the help of the hierarchical structure. As the first example, we apply the machinery to the case of 
cylinder. The GF can be concisely given in terms of the variables introduced in $[9,10]$.

The paper is organized as follows. In Sec. II, description of IT and MG in terms of the KdV hierarchy and Virasoro constraints is summarized and clarified: Sec. II A is for the closed Riemann surfaces and Sec. II B is for the Riemann surfaces with boundaries (open Riemann surfaces). In Sec. III, GF of BMG with $\mu_{B}$ parameter is analyzed in terms of open KdV hierarchy with Euler characteristic expansion. In Sec. IV, we find GF of OIT in $s$ space using the Laplace transform from GF of BMG in $\mu_{B}$ space. In this process, one has to find a way to choose the right solution of string equation. We provide a formula for the disk GF in $s$ space with dependence on all the $\mathrm{KdV}$ parameters, generating the explicit formula of [6] where the first of the $\mathrm{KdV}$ parameters being turned off. We further derive a formula for the GF on a fluctuating cylinder and check if it reproduces a result given in a form of a power series generated by perturbative analysis [11]. This demonstrates that our approach is powerful and simplified compared to preceding approaches. Section V is the summary and discussion.

\section{MINIMAL GRAVITY VS INTERSECTION THEORY}

\section{A. Riemann surfaces without boundary}

Minimal quantum gravity of Lee-Yang series $M(2,2 p+1)$ on the closed Riemann surface is described either by the Liouville field theory coupled to conformal matter [12] or by the scaling limit of one-matrix model $[13,14]$. Its generating function (free energy) has natural genus expansion

$$
\mathcal{F}^{c}=\sum_{g=0}^{\infty} \lambda^{2 g-2} \mathcal{F}_{(g)}^{c},
$$

where $\lambda$ is the genus expansion parameter. Two approaches led to the different expressions for the GF, which are believed to be related to each other by the so-called resonance transformations $[15,16]$. Below we will work with the matrix model description, which has a much clearer relation to the integrable hierarchies. The GF $\mathcal{F}^{c}$ depends on the multiple $\mathrm{KdV}$ parameters $\tau_{0}, \tau_{1}, \ldots, \tau_{p-1}$, and it is convenient to introduce the function

$$
u \equiv \frac{\partial^{2} \mathcal{F}^{c}}{\partial \tau_{0}^{2}}
$$

The matrix model formulation allows us to construct the GF, $\mathcal{F}^{c}$, dependent on the infinitely many descendent variables. However, we do not consider this opportunity below.

The flow equations of $u$ along the KdV parameter directions constitute the KdV hierarchy [17-20]. The $\mathrm{KdV}$ hierarchy and the string equation satisfied by the GF of MG can be represented as

$$
\begin{aligned}
\frac{1}{\lambda^{2}} \frac{2 n+1}{2} \frac{\partial^{3} \mathcal{F}^{c}}{\partial \tau_{0}^{2} \partial \tau_{n}}= & \frac{\partial^{2} \mathcal{F}^{c}}{\partial \tau_{0}^{2}} \frac{\partial^{3} \mathcal{F}^{c}}{\partial \tau_{0}^{2} \partial \tau_{n-1}}+\frac{1}{2} \frac{\partial^{3} \mathcal{F}^{c}}{\partial \tau_{0}^{3}} \frac{\partial^{2} \mathcal{F}^{c}}{\partial \tau_{0} \partial \tau_{n-1}} \\
& +\frac{1}{8} \frac{\partial^{5} \mathcal{F}^{c}}{\partial \tau_{0}^{4} \partial \tau_{n-1}} \\
0= & \sum_{n \geq 0} \tau_{n+1} \frac{\partial \mathcal{F}^{c}}{\partial \tau_{n}}+\frac{\tau_{0}^{2}}{2 \lambda^{2}}
\end{aligned}
$$

where $\tau_{p+1}=1$ and $\tau_{k}=0$ for $k>p+1$.

On a fluctuating sphere, the flows are described by the dispersionless limit of the KdV hierarchy (3), which has the simple form

$$
\frac{\partial^{3} \mathcal{F}_{(0)}^{c}}{\partial \tau_{n} \partial \tau_{0}^{2}}=\frac{\partial v}{\partial \tau_{n}}=\frac{v^{n}}{n !} \frac{\partial v}{\partial \tau_{0}} \quad \text { for } 1 \leq n \leq p-1 .
$$

Here $\mathcal{F}_{(0)}^{c}$ is the GF on the sphere, and it is best described in terms of $A_{1}$ Frobenius manifold [21,22] (see also [23,24] for a dual description in terms of $A_{2 p}$ ) whose coordinate is identified with the second derivative of the GF,

$$
v \equiv \frac{\partial^{2} \mathcal{F}_{(0)}^{c}}{\partial \tau_{0}^{2}} .
$$

The string equation can be reduced to the polynomial form

$\mathcal{P}(\tau, v)=0 ; \quad \mathcal{P}(\tau, v) \equiv \sum_{m=0}^{p-1} \tau_{m} \frac{v^{m}}{m !}+\frac{v^{p+1}}{(p+1) !}$.

This equation can be obtained if one takes the second derivative of (4) with respect to $\tau_{0}$, uses the dispersionlees KdV hierarchy (5), and integrates the result over $\tau_{0}$.

It is noted that GF of MG is constructed on the fluctuating sphere in [25] for the Lee-Yang model using both of the results of Liouville field theory and matrix model. This result is extended in [16] to the Lee-Yang series

$$
\mathcal{F}_{(0)}^{c}=\frac{1}{2} \int_{0}^{w} \mathcal{P}^{2}(v) d v,
$$

where $\mathcal{P}(v)$ is the string polynomial and $w$ is a proper solution of the string equation of the polynomial form (7). One can check easily that $\mathcal{F}_{(0)}^{c}$ satisfies the $\mathrm{KdV}$ hierarchy and the string equation. GF of MG is further constructed up to genus 3 in [3], and these contributions are also found to satisfy the KdV hierarchy.

In [1], it was conjectured that two-dimensional gravity is related to the intersection theory on the moduli space of Riemann surfaces. The GF of IT depends on the infinitely many parameters, $t=\left(t_{0}, t_{1}, \cdots\right)$, playing the role of the coupling constants of the gravitational descendants in topological gravity. From this physical identification of 
different two-dimensional gravity models, Witten concluded that the all genera GF of IT, $F^{c}$, also satisfies the $\mathrm{KdV}$ hierarchy and the string equation

$$
\begin{aligned}
\frac{1}{\lambda^{2}} \frac{2 n+1}{2} \frac{\partial^{3} F^{c}}{\partial t_{0}^{2} \partial t_{n}}= & \frac{\partial^{2} F^{c}}{\partial t_{0}^{2}} \frac{\partial^{3} F^{c}}{\partial t_{0}^{2} \partial t_{n-1}}+\frac{1}{2} \frac{\partial^{3} F^{c}}{\partial t_{0}^{3}} \frac{\partial^{2} F^{c}}{\partial t_{0} \partial t_{n-1}} \\
& +\frac{1}{8} \frac{\partial^{5} F^{c}}{\partial t_{0}^{4} \partial t_{n-1}}, \\
\frac{\partial F^{c}}{\partial t_{0}} & =\sum_{n \geq 0} t_{n+1} \frac{\partial F^{c}}{\partial t_{n}}+\frac{t_{0}^{2}}{2 \lambda^{2}} .
\end{aligned}
$$

Here and from now on, we distinguish the notation of GF, $\mathcal{F}$ for MG, and $F$ for IT. The conjecture was proved in [2] by identifying of the GF of IT with the matrix integral over $N \times N$ Hermitian matrix $X$ with the cubic potential

$$
e^{F^{c}} \propto \int[d X] e^{-\frac{1}{\lambda} \operatorname{Tr}\left(\frac{X^{3}}{6}-\frac{\Lambda^{2} X}{2}\right)},
$$

with the condition $F^{c}(t=0)=0$. For this Kontsevich matrix integral representation, the set of KdV parameters is given by the Miwa variables

$$
t_{k}=\lambda(2 k-1) ! ! \operatorname{Tr} \Lambda^{-2 k-1},
$$

assuming $N$ is sufficiently large.

It is known that the $\mathrm{KdV}$ hierarchy and string equation imply the Virasoro constraints $[7,8]$,

$$
L_{n} e^{F^{c}}=0 \quad \text { for } n \geq-1 .
$$

The Virasoro generators are

$$
\begin{gathered}
L_{-1}=\sum_{i \geq 0}\left(t_{i}-\delta_{i, 1}\right) \frac{\partial}{\partial t_{i-1}}+\frac{t_{0}^{2}}{2 \lambda^{2}}, \\
L_{0}=\sum_{i \geq 0} \frac{2 i+1}{2}\left(t_{i}-\delta_{i, 1}\right) \frac{\partial}{\partial t_{i}}+\frac{1}{16},
\end{gathered}
$$

and for $n>0$,

$$
\begin{aligned}
L_{n}= & \sum_{i \geq 0} \frac{(2 i+2 n+1) ! !}{2^{n+1}(2 n-1) ! !}\left(t_{i}-\delta_{i, 1}\right) \frac{\partial}{\partial t_{i+n}} \\
& +\frac{\lambda^{2}}{2} \sum_{i \geq 0}^{n-1} \frac{(2 i+1) ! !(2 n-2 i-1) ! !}{2^{n+1}} \frac{\partial^{2}}{\partial t_{i} \partial t_{n-i-1}} .
\end{aligned}
$$

The Virasoro constraints can also be derived from the Kontsevich matrix integral [26-28].

From the comparison of the KdV hierarchy and the string equation of MG (3) and (4) and those of IT (9) and (10) one can conclude, that they coincide after an identification of all variables $\tau_{n}$ with $t_{n}$ except for $n=1$. Namely, $t_{1}$ is identified with $\tau_{1}$ shifted by a constant, $t_{1}=\tau_{1}+1$. This is a wellknown dilaton shift. Let us stress that, while the equation satisfied by the GF of IT and that of MG are almost the same, the solutions do not coincide. In particular, the role of $\mathrm{KdV}$ parameters in two models differs dramatically. First, $\tau_{n}$ in MG couples to the gravitation primary operator of Lee-Yang series. On the other hand, $t_{n}$ for $n>0$ in IT couples to gravitational descendant operator.

Second, more important is the role of $\tau_{p-1}$ of $\mathrm{MG}$ and $t_{0}$ of IT. In MG approach, the cosmological constant $\mu$ should be present and all the physical quantities in MG are equipped with the GSD, which counts the power of $\mu$ [12]. It is known $[15,25]$ that $\tau_{p-1}$ plays the role of $\mu$. Other $\mathrm{KdV}$ parameters $\tau_{n}(n<p-1)$ are considered as deformation parameters. Before the deformation, the string equation of the polynomial form (7)

$$
\tau_{p-1} \frac{v^{p-1}}{(p-1) !}+\frac{v^{p+1}}{(p+1) !}=0
$$

has a nontrivial solution $v \propto \sqrt{\mu}$ and namely this solution describes MG. This shows that, in general, GF of MG is nonanalytic in $\tau_{p-1}$, as GF is given in powers of $v$ [see, e.g., (8)] and GSD is given by a fractional number.

It can be shown that the GF of MG is scale free. Note that in (7) the coefficient of the term $v^{p+1}$ is assumed to be scale free and can be normalized to 1 . Since GSD of $v$ is $1 / 2$, GSD of $\mathcal{P}(\tau, v)$ is $(p+1) / 2$ and GSD of the deformation parameters $\tau_{k}$ is $(p+1-k) / 2$. According to (8), GSD of GF on sphere is $(2 p+3) / 2$. In addition, the genus expansion parameter $\lambda^{2}$ in (1) has nonvanishing GSD, namely, $(2 p+3) / 2$.

To compare the IT with the MG, we can put $t_{n}=0$ $(n \geq p)$ in the GF, which restricts to the subspace with the finite number of $\mathrm{KdV}$ parameters $t=\left(t_{0}, t_{1}, \cdots t_{p-1}\right)$. Then, the string polynomial for IT is obtained from (7) by the above described between $\tau$ and $t$,

$$
P(t, v)=\sum_{m=0}^{p-1} t_{m} \frac{v^{m}}{m !}-v,
$$

where the linear power of $v$ is added due to the $t_{1}$ shift. In this case, $t_{0}$ becomes the basic parameter and the others are treated as deformation parameters, so that the undeformed solution is $v=t_{0}$. One can show that the string polynomial (18) is consistent with the KdV hierarchy (9) and the string equation (10) to the lowest order in $\lambda$.

The solution of this string equation, corresponding to IT, is completely perturbative in $t_{k}$ 's. Namely, it is a power series of all the KdV parameters $t_{k}$ so it has a regular limit when all of them, including $t_{0}$, go to zero. In a certain sense, the GF of IT can be considered as a "universal" GF for the whole Lee-Yang series, starting from $M(2,1)$ model, 
adding proper number of variables and allowing analytic continuation on the solution space [3].

Like in MG, one can introduce the SD to IT: in (18), $t_{1}$ is assumed to be a scale-free parameter. Therefore, it is natural to define $\mathrm{SD}$ as the power of $t_{0}$, the basic scale parameter. This shows that SD of $v$ is 1 and therefore, $\mathrm{SD}$ of $P(t, v)$ is assigned to be 1 and SD of the deformation parameters $t_{k}$ is $1-k$. Note that $v=t_{0}$ before deformation, and that SD of GF on sphere is 3 (from the definition $v=\partial^{2} F_{(0)}^{c} / \partial t_{0}^{2}$ ), so is the SD of the genus expansion parameter $\lambda^{2}$.

\section{B. Riemann surfaces with boundaries}

Recently, the intersection theory on the moduli spaces of the Riemann surfaces with boundaries was developed by Pandharipande et al. [6]; see also [29,30]. They have described the GF $F^{o}$ for the open intersection numbers

$$
\int_{\overline{\mathcal{M}}_{\bar{g}, k, l}} \psi_{1}^{a_{1}} \psi_{2}^{a_{2}} \ldots \psi_{l}^{a_{l}}
$$

given by the integrals of the products of the first Chern classes $\psi_{i}$ of the cotangent line bundles over the compactification $\overline{\mathcal{M}}_{\bar{g}, k, l}$ of the moduli space of Riemann surfaces with boundaries. They also constructed explicitly the leading contribution to this GF, given by the disk geometry, and explained how to make the naive description (19) precise for the higher geometries. The integral is nonvanishing only if dimension of $\overline{\mathcal{M}}_{\bar{g}, k, l}$,

$$
\operatorname{dim}_{\mathbb{R}} \mathcal{M}_{\bar{g}, k, l}=3 \bar{g}-3+k+2 l,
$$

coincides with the degree of the integrand

$$
3 \bar{g}-3+k+2 l=\sum_{j=1}^{l} 2 a_{j},
$$

and the stability condition $2 \bar{g}-2+k+2 l>0$ is satisfied.

The GF, which depends on the KdV parameters $t_{k}$ and an additional parameter $s$, associated with the insertion of the marked points on the boundary, has a natural topological expansion

$$
F^{o}=\sum_{\bar{g}=0}^{\infty} \lambda^{\bar{g}-1} F_{(\bar{g})}^{o} .
$$

Here $\bar{g}$ is the genus of the doubled Riemann surface. This expansion can be interpreted as the Euler characteristic expansion

$$
F^{o}=\sum_{\chi \leq 1} \lambda^{-\chi} F_{(\chi)}^{o},
$$

where $\chi=2-2 g-k$ is given in terms of the number of handles $(g \geq 0)$ and the number of boundaries $(k \geq 1)$ of the Riemann surface with boundaries, and is related to $\bar{g}$ as $\bar{g}=1-\chi$. Hereafter, we call the Euler characteristics expansion the $\bar{g}$ expansion.

The authors of [6] also suggested a generalization of the Virasoro constraints (13) for the open case,

$$
B_{n} e^{F^{c}+F^{o}}=0 \text { for } n \geq-1,
$$

where

$$
B_{n}=L_{n}+\lambda^{n} s \frac{\partial^{n+1}}{\partial s^{n+1}}+\frac{3 n+3}{4} \lambda^{n} \frac{\partial^{n}}{\partial s^{n}} .
$$

In particular, for $n=-1$ with the help of the string equation (10), Eq. (24) reduces to the open string equation

$$
\frac{\partial F^{o}}{\partial t_{0}}=\sum_{n \geq 0} t_{n+1} \frac{\partial F^{o}}{\partial t_{n}}+\frac{s}{\lambda}
$$

An open version of the KdV hierarchy, satisfied by the open GF, was also introduced in [6]

$$
\begin{aligned}
\frac{2 n+1}{2} \frac{\partial F^{o}}{\partial t_{n}}= & \lambda \frac{\partial F^{o}}{\partial s} \frac{\partial F^{o}}{\partial t_{n-1}}+\lambda \frac{\partial^{2} F^{o}}{\partial s \partial t_{n-1}}+\frac{\lambda^{2}}{2} \frac{\partial F^{o}}{\partial t_{0}} \frac{\partial^{2} F^{c}}{\partial t_{0} \partial t_{n-1}} \\
& -\frac{\lambda^{2}}{4} \frac{\partial^{3} F^{c}}{\partial t_{0}^{2} \partial t_{n-1}} .
\end{aligned}
$$

While the relation of the open $\mathrm{KdV}$ equations to the integrable hierarchies remains unclear, it was proven by Buryak in [31], that the open $\mathrm{KdV}$ hierarchy has a unique solution with the given initial conditions, and this solution satisfies the open Virasoro constraints (24). Buryak also found an additional $s$-flow equation, which is consistent with the open KdV hierarchy

$$
\frac{\partial F^{o}}{\partial s}=\lambda\left\{\frac{1}{2}\left(\frac{\partial F^{o}}{\partial t_{0}}\right)^{2}+\frac{1}{2} \frac{\partial^{2} F^{o}}{\partial t_{0}^{2}}+\frac{\partial^{2} F^{c}}{\partial t_{0}^{2}}\right\} .
$$

Having in mind the connection between IT and MG in the closed case, outlined in Sec. II 1, it is natural to expect a similar connection for the case with boundaries. The GF of BMG has the $\bar{g}$ expansion (22),

$$
\mathcal{F}^{o}=\sum_{\bar{g}=0}^{\infty} \lambda^{\bar{g}-1} \mathcal{F}_{(\bar{g})}^{o} .
$$

However, the description of the OIT looks completely different from that of the BMG. Namely, boundary effects in $\mathrm{BMG}$ are described by the boundary cosmological constant $\mu_{B}$, whose nature essentially differs from that of the boundary marked point insertion parameter $s$ of the OIT.

A clue to the relation between two pictures can be seen from the comparison of the equations, satisfied by the leading terms of the $\bar{g}$ expansion, that is GFs on the disk. For the BMG on the disk, $\mathcal{F}_{(0)}^{o}$, one has [15] 


$$
\mathcal{F}_{(0)}^{o}\left(\tau, \mu_{B}\right)=\frac{i}{\sqrt{2 \pi}} \int_{0}^{\infty} \frac{d l}{l^{3 / 2}} e^{-l \mu_{B}} \int_{\tau_{0}}^{\infty} d x e^{-l v(x)}
$$

Here $v(x)$ is a function of $x$, which is governed by the string equation of the polynomial form (7), with $\tau_{0}$ substituted by $x$. The GF in (30) satisfies the following equation [4]:

$$
\frac{2 n+1}{2} \frac{\partial \mathcal{F}_{(0)}^{o}}{\partial \tau_{n}}=-\mu_{B} \frac{\partial \mathcal{F}_{(0)}^{o}}{\partial \tau_{n-1}}+\frac{1}{2} \frac{\partial \mathcal{F}_{(0)}^{o}}{\partial \tau_{0}} \frac{\partial^{2} \mathcal{F}_{(0)}^{c}}{\partial \tau_{0} \partial \tau_{n-1}}
$$

for $1 \leq n \leq p-1$. This equation can be easily derived if one notes that the multiplication of the integrand of (30) by $-\mu_{B}$ can be replaced by a derivative with respect to $l$. Details are given in the Appendix A. This functional relation is similar to the open $\mathrm{KdV}(27)$ at $\bar{g}=0: \mathcal{F}_{(0)}^{o}$ corresponds to $F_{(0)}^{o}$, and $\partial F_{(0)}^{o} / \partial s$ is replaced by $-\mu_{B}$, which is assumed to be independent of $\tau_{n}$.

This observation allows us to conjecture that the $s$ and $\mu_{B}$ pictures are related by the Laplace (or Fourier) transform [5]

$$
e^{\mathcal{F}^{o}(s)}=\frac{1}{\sqrt{2 \pi \lambda}} \int d \mu_{B} e^{-\frac{s \mu_{B}}{\lambda}} e^{\mathcal{F}^{o}\left(\mu_{B}\right)}
$$

with the inverse transform

$$
e^{\mathcal{F}^{o}\left(\mu_{B}\right)}=\frac{1}{\sqrt{2 \pi \lambda}} \int d s e^{\frac{s \mu_{B}}{\lambda}} e^{\mathcal{F}^{o}(s)}
$$

To avoid confusion, we indicate explicitly the variable $s$ or $\mu_{B}$. Here we continue to use the notation $\mathcal{F}$ after the Laplace transform and, depending on the context, we assume it to depend on the $\mathrm{KdV}$ variables $\tau$ or $t$. The reason is that we expect this relation still to be valid beyond the proper parameter range of the OIT of [6]. As we will show in the following section, to get the GF of OITone has to choose very particular $\mathcal{F}^{o}\left(\mu_{B}\right)$, which corresponds to a certain topological branch of BMG.

The Laplace transform in (32) and (33) can be interpreted with the saddle point method for the large values of $\lambda$. Then the critical values of $\mu_{B}$ and $s$ in (32) and (33) satisfy, respectively,

$$
s=\frac{\partial \mathcal{F}_{(0)}^{o}\left(\mu_{B}\right)}{\partial \mu_{B}}
$$

and

$$
\mu_{B}=-\frac{\partial \mathcal{F}_{(0)}^{o}(s)}{\partial s},
$$

which allows to express $\mu_{b}$ in terms of $s$ and $t$ or $\tau$ and vice versa. Thus, the disk amplitudes are related by the Legendre transform

$$
\mathcal{F}_{(0)}^{o}(s)=\mathcal{F}_{(0)}^{o}\left(\mu_{B}\right)-s \mu_{B} .
$$

The next orders of the $\bar{g}$ expansion can be obtained by computation of the Gaussian integral with perturbation, in particular

$$
\mathcal{F}_{(1)}^{o}(s)=\mathcal{F}_{(1)}^{o}\left(\mu_{B}\right)-\frac{1}{2} \log \left(\frac{\partial^{2} \mathcal{F}_{(0)}^{o}\left(\mu_{B}\right)}{\partial \mu_{B}^{2}}\right),
$$

where the expression for $\mu_{B}$ in (35) is used.

Another, but essentially equivalent, version of the Laplace transform of GF of OIT was considered in [29]. It was shown that after the Laplace transform the GF of OIT coincides with the Baker-Akhiezer function of the Kontsevich-Witten tau-function of the KdV hierarchy,

$e^{F^{o}(t, z)}=z^{-1 / 2} e^{\lambda^{-1} \Sigma_{k \geq 0}\left(t_{k}-\delta_{k, 1}\right) \frac{2^{2 k+1}}{(2 k+1) ! !}} e^{F^{c}\left(t_{k}-\lambda \frac{(2 k-1) ! !}{z^{k k+1}}\right)-F^{c}(t)}$,

where $z$ is to be identified with $i \sqrt{2 \mu_{B}}$. In Appendix B, we compare the first term of this identity with our simplest result in Sec. IV 1. The same relation between the GF of open and closed versions of MG, which is probably the simplest example of the more fundamental relation between open and closed theories, was obtained in [32]. This demonstrates that the Laplace transform (33) indeed provides a correct way to introduce the boundary cosmological constant into the OIT. Below, we describe explicit computations, which also support this claim.

The conjectural relation through the Laplace transform allows us to translate the properties of the GF from $s$ to $\mu_{B}$ pictures and back. In particular, the Virasoro constraints

$$
\mathcal{C}_{n} e^{\mathcal{F}^{c}+\mathcal{F}^{o}\left(\mu_{B}\right)}=0 \quad \text { for } n \geq-1,
$$

where [5]

$$
\mathcal{C}_{n}=\mathcal{L}_{n}+\left(-\mu_{B}\right)^{n}\left(-\mu_{B} \frac{\partial}{\partial \mu_{B}}-\frac{n+1}{4}\right),
$$

with $\mathcal{L}_{n}$ being $L_{n}$, replacing $t_{i}$ with $\tau_{i}$ except $t_{1} \rightarrow \tau_{1}+1$, can be obtained by the Laplace transform of Virasoro generators (25) for the cases, where the GF depends on the infinite set of the KdV variables [29,33,34].

Similarly, for the open KdV hierarchy in $\mu_{B}$ space, we have

$$
\begin{aligned}
\frac{2 n+1}{2} \frac{\partial \mathcal{F}^{o}}{\partial \tau_{n}}= & -\mu_{B} \frac{\partial \mathcal{F}^{o}}{\partial \tau_{n-1}}+\frac{\lambda^{2}}{2} \frac{\partial \mathcal{F}^{o}}{\partial \tau_{0}} \frac{\partial^{2} \mathcal{F}^{c}}{\partial \tau_{0} \partial \tau_{n-1}} \\
& -\frac{\lambda^{2}}{4} \frac{\partial^{3} \mathcal{F}^{c}}{\partial \tau_{0}^{2} \partial \tau_{n-1}} \quad \text { for } n \geq 1 .
\end{aligned}
$$

The open string equation becomes 


$$
0=\sum_{n \geq 0} \tau_{n+1} \frac{\partial \mathcal{F}^{o}}{\partial \tau_{n}}+\frac{\partial \mathcal{F}^{o}}{\partial \mu_{B}}
$$

In addition, the $s$-flow equation (28) has a new form in the $\mu_{B}$ space

$$
-\mu_{B}=\lambda\left\{\frac{1}{2}\left(\frac{\partial \mathcal{F}^{o}}{\partial \tau_{0}}\right)^{2}+\frac{1}{2} \frac{\partial^{2} \mathcal{F}^{o}}{\partial \tau_{0}^{2}}+\frac{\partial^{2} \mathcal{F}^{c}}{\partial \tau_{0}^{2}}\right\}
$$

which we call boundary condition equation (BCE). The BCE provides a simple and consistent check of the GF in the presence of the boundary and the boundary cosmological constant plays a role of the boundary condition.

\section{GENERATING FUNCTION OF MINIMAL GRAVITY WITH BOUNDARIES}

\section{A. Generating function on a disk}

According to one-matrix model approach, the continuum limit of the matrix variable is described by a differential operator $\hat{Q}_{2}=-\partial_{x}^{2}+u(x)$, where $u$ is given by (2) $[17,18]$, and the GF of BMG can be expressed in terms of $u$, obtained from GF of MG without boundary. For the GF on a disk, one may use the dispersionless limit (neglecting derivatives) of $\hat{Q}_{2}$, the second order polynomial in $y ; Q_{2}=y^{2}+v(x)$.

The GF on a disk has the integral representation (30) with the proper normalization [15]

$$
\begin{aligned}
\mathcal{F}_{(0)}^{o}\left(\tau, \mu_{B}\right) & =\frac{i}{\sqrt{2 \pi^{2}}} \int_{0}^{\infty} \frac{d l}{l} e^{-l \mu_{B}} \int_{\tau_{0}}^{\infty} d x \int_{\mathbb{R}} d y e^{-1\left(y^{2}+v(x)\right)} \\
& =\frac{i}{\sqrt{2 \pi}} \int_{0}^{\infty} \frac{d l}{l^{3 / 2}} e^{-l \mu_{B}} \int_{\tau_{0}}^{\infty} d x e^{-l v(x)}
\end{aligned}
$$

and is given symbolically by $\left\langle\operatorname{Tr} \log \left(\mu_{B}+Q_{2}\right)\right\rangle$, which is straightforwardly extendable to incorporate multiple boundaries and to impose boundary conditions [35]. In III A, $v(x)$ is the solution of the string polynomial equation (7) with $\tau_{0}$ replaced by $x$. As is discussed in the previous section, there are $p+1$ solutions to this equation, and to get the GF of $\mathrm{BMG}$, one has to take the solution $v(x) \propto \sqrt{-\tau_{p-1}}$ when all the parameters switched off except $\tau_{p-1}(\propto \mu)$.

This GF satisfies the lowest order of the open $\mathrm{KdV}$ hierarchy in $\mu_{B}$ space (31) as we show in Appendix A. The open string equation (42) in the lowest order in $\lambda$ trivially follows from the string polynomial equation (7). In addition, one can show that the lowest order of the BCE (43)

$$
-\mu_{B}=\frac{1}{2}\left(\frac{\partial \mathcal{F}_{(0)}^{o}}{\partial \tau_{0}}\right)^{2}+\frac{\partial^{2} \mathcal{F}_{(0)}^{c}}{\partial \tau_{0}^{2}}
$$

is also satisfied if one notes that

$$
\frac{\partial \mathcal{F}_{(0)}^{o}}{\partial \tau_{0}}=-i \sqrt{\frac{\mu_{B}+w}{2 \pi}} \Gamma\left(-\frac{1}{2}\right)=\sqrt{2} i \sqrt{\mu_{B}+w}
$$

where $w=v\left(\tau_{0}\right)$ denotes the relevant solution of $\mathcal{P}(\tau, v)=0$.

To simplify the expression, one can change the integration variable $x$ into $v$ if the string polynomial equation for $\mathcal{P}(\tau, v)$ in $(7)$ is used,

$$
\mathcal{F}_{(0)}^{o}\left(\tau, \mu_{B}\right)=-\frac{i}{\sqrt{2 \pi}} \int_{0}^{\infty} \frac{d l}{l^{3 / 2}} e^{-l \mu_{B}} \int_{w}^{\infty} d v \mathcal{P}^{(1)}(\tau, v) e^{-l v}
$$

Here $\mathcal{P}^{(1)}(\tau, v)=d \mathcal{P} / d v$ plays the role of the Jacobian factor $d x / d v=-d \mathcal{P} / d v$. It should be emphasized that the integration variable $v$ is independent of $\tau_{n}$. After the integration by parts in $v$, one gets

$$
\mathcal{F}_{(0)}^{o}\left(\tau, \mu_{B}\right)=-\frac{i}{\sqrt{2 \pi}} \int_{0}^{\infty} \frac{d l}{l^{1 / 2}} e^{-l \mu_{B}} \int_{w}^{\infty} d v \mathcal{P}(\tau, v) e^{-l v}
$$

where we use $\mathcal{P}(\tau, w)=0$. Therefore, one has

$$
\mathcal{F}_{(0)}^{o}\left(\tau, \mu_{B}\right)=\sum_{n} \tau_{n}\left\langle O_{n}\right\rangle_{\mathrm{disk}}
$$

where, due to $\mathcal{P}(\tau, w)=0$, we have an identity

$$
\left\langle O_{n}\right\rangle_{\mathrm{disk}}=\frac{\partial \mathcal{F}_{(0)}^{o}}{\partial \tau_{n}}=-\frac{i}{\sqrt{2 \pi}} \int_{0}^{\infty} \frac{d l}{l^{1 / 2}} e^{-l \mu_{B}} \int_{w}^{\infty} d v \frac{v^{n}}{n !} e^{-l v}
$$

and

$$
0=\sum_{n} \tau_{n} \frac{\partial\left\langle O_{n}\right\rangle_{\text {disk }}}{\partial \tau_{m}}
$$

The correlation functions $\left\langle O_{n}\right\rangle_{\text {disk }}$ depend only on $w$ and $\mu_{B}$.

To evaluate the integrals in (50), we scale $v=w \eta$ and put the scale-free monomial $\eta^{n}$ as a linear combination of the Legendre polynomials $P_{k}$,

$$
\eta^{n}=\sum_{k=n, n-2, \ldots \geq 0}(2 k+1) n ! a_{n, k} P_{k}(\eta)
$$

where

$$
a_{n, k}=\frac{1}{2^{(n-k) / 2}((n-k) / 2) !(n+k+1) ! !} .
$$

Then the integration over $\eta$ is given as the modified Bessel function of the second kind $K_{n}$, 


$$
\int_{1}^{\infty} d \eta e^{-w l \eta} P_{k}(\eta)=\sqrt{\frac{2}{\pi w l}} K_{1 / 2+k}(w l) .
$$

Furthermore, the integration over $l$ is performed (after analytic continuation if necessary) to give

$$
\int_{0}^{\infty} \frac{d l}{l} e^{-l \mu_{B}} K_{1 / 2+k}(w l)=\frac{2 \pi(-1)^{k+1}}{2 k+1} \cosh ((1 / 2+k) \theta) .
$$

Here we put $\mu_{B}=w \cosh (\theta)$. As a result, (50) is given in terms of the Chebyshev polynomial $T_{n}(\cosh (x))=$ $\cosh (n x)$,

$$
\begin{aligned}
\left\langle O_{n}\right\rangle_{\text {disk }}= & -i w^{n+1 / 2}(-1)^{n+1} \\
& \times \sum_{k=n, n-2, \ldots \geq 0} a_{n, k} T_{2 k+1}(\cosh (\theta / 2)) .
\end{aligned}
$$

It is noted that $\left\langle O_{n}\right\rangle_{\text {disk }}$ in general depends on the $\mathrm{KdV}$ parameters since $w$ and $\theta$ are functions of $\mathrm{KdV}$ parameters. However, on-shell $w$ and $\theta$ reduce to certain constant values, $w \propto \sqrt{\mu}$ and $\cosh (\theta) \propto \mu_{B} / \sqrt{\mu}$.

\section{B. Higher $\bar{g}$ expansion}

The open KdV hierarchy (41) and the open string equation (42) allow one to further evaluate the higher $\bar{g} \geq$ 1 contributions using the $\bar{g}$ expansion

$$
\begin{aligned}
\frac{2 n+1}{2} \frac{\partial \mathcal{F}_{(\bar{g})}^{o}}{\partial \tau_{n}}= & -\mu_{B} \frac{\partial \mathcal{F}_{(\bar{g})}^{o}}{\partial \tau_{n-1}}+\frac{1}{2} \sum_{\bar{g}_{1}+2 \bar{g}_{2}=\bar{g}}\left(\frac{\partial \mathcal{F}_{\left(\bar{g}_{1}\right)}^{o}}{\partial \tau_{0}} \frac{\partial^{2} \mathcal{F}_{\left(\bar{g}_{2}\right)}^{c}}{\partial \tau_{0} \partial \tau_{n-1}}\right) \\
& -\frac{1}{4} \frac{\partial^{3} \mathcal{F}_{((\bar{g}-1) / 2)}^{c}}{\partial \tau_{0}^{2} \partial \tau_{n-1}} \\
0 & =\sum_{n \geq 0} \tau_{n+1} \frac{\partial \mathcal{F}_{(\bar{g})}^{o}}{\partial \tau_{n}}+\frac{\partial \mathcal{F}_{(\bar{g})}^{o}}{\partial \mu_{B}}
\end{aligned}
$$

Here and below the term $\mathcal{F}_{((\bar{g}-1) / 2)}^{c}$ is present only when $\bar{g}$ is odd. In addition, BCE (43) shows that higher $\bar{g} \geq 1$ equation becomes linear in $\mathcal{F}_{(\bar{g})}^{o}$,

$0=\sum_{\bar{g}_{1}+\bar{g}_{2}=\bar{g}} \frac{1}{2} \frac{\partial \mathcal{F}_{\left(\bar{g}_{1}\right)}^{o}}{\partial \tau_{0}} \frac{\partial \mathcal{F}_{\left(\bar{g}_{2}\right)}^{o}}{\partial \tau_{0}}+\frac{1}{2} \frac{\partial^{2} \mathcal{F}_{(\bar{g}-1)}^{o}}{\partial \tau_{0}^{2}}+\frac{\partial^{2} \mathcal{F}_{(\bar{g} / 2)}^{c}}{\partial \tau_{0}^{2}}$.

The $\bar{g}$ expansion shows that higher $\bar{g}$ contribution, more precisely, its first derivatives, is given in terms of lower $\bar{g}$ solution. Therefore, GF for each $\bar{g}$ can be obtained from $\mathcal{F}_{(0)}^{o}\left(\tau, \mu_{B}\right)$, which satisfies the lowest order nonlinear equation (45).

One can obtain the GF for $\bar{g}=1$ if one uses the BCE (59) which simplifies for $\bar{g}=1$ as the following:

$$
\begin{aligned}
0 & =\frac{\partial \mathcal{F}_{(0)}^{o}}{\partial \tau_{0}} \frac{\partial \mathcal{F}_{(1)}^{o}}{\partial \tau_{0}}+\frac{1}{2} \frac{\partial^{2} \mathcal{F}_{(0)}^{o}}{\partial \tau_{0}^{2}} \\
& =\frac{\partial \mathcal{F}_{(0)}^{o}}{\partial \tau_{0}} \frac{\partial}{\partial \tau_{0}}\left(\mathcal{F}_{(1)}^{o}+\frac{1}{2} \log \left(\frac{\partial \mathcal{F}_{(0)}^{o}}{\partial \tau_{0}}\right)\right) .
\end{aligned}
$$

The solution has the form

$$
\mathcal{F}_{(1)}^{o}=-\frac{1}{2} \log \left(\frac{\partial \mathcal{F}_{(0)}^{o}}{\partial \tau_{0}}\right),
$$

where $\tau_{0}$-independent but $\tau_{n>0}$-dependent contribution turns out to vanish except the trivial constant [5].

This solution can be compared with the GF of BMG from the matrix model computation on cylinder $\mathcal{F}_{(1)}^{o} \propto$ $\left\langle\left(\operatorname{Tr} \log \left(M+\mu_{B}\right)\right)^{2}\right\rangle_{c}$, where the subscript $c$ denotes the connected part and $M$ is the Hermitian matrix. In the continuum limit, one has to replace $M$ by $Q_{2}$ to have the form [18]

$$
\begin{aligned}
\mathcal{F}_{(1)}^{o}= & \left(\frac{i}{\sqrt{2 \pi^{2}}}\right)^{2} \int_{\tau_{0}}^{\infty} d x_{1} \int_{-\infty}^{\tau_{0}} d x_{2} \int_{0}^{\infty} \frac{d l_{1} d l_{2}}{l_{1} l_{2}} \\
& \times\left\langle x_{1}\left|e^{-l_{1}\left(\mu_{B}+w-\partial^{2}\right)}\right| x_{2}\right\rangle\left\langle x_{2}\left|e^{-l_{2}\left(\mu_{B}+w-\partial^{2}\right)}\right| x_{1}\right\rangle,
\end{aligned}
$$

where the integration range of $x_{1}$ does not overlap with that of $x_{2}$ because of the connected part. In addition, $v(x)$ in $Q_{2}$ is replaced by $w$ because derivative of $v$ does not contribute to the cylindrical contribution [15]. One may evaluate the integral easily, by inserting the identity $1=$ $\int_{-\infty}^{\infty} d p_{a}\left|p_{a}\right\rangle\left\langle p_{a}\right|$, with $\left\langle p_{a} \mid x_{i}\right\rangle=e^{i p_{a} x_{i}}$, and performing the $p_{a}$ integral (Gaussian integral), the matrix element is evaluated as

$$
\left\langle x_{i}\left|e^{l_{i} \partial^{2}}\right| x_{j}\right\rangle=\sqrt{\frac{\pi}{l_{i}}} e^{-\frac{\left(x_{i}-x_{j}\right)^{2}}{l_{i}}} .
$$

This shows that [15]

$\mathcal{F}_{(1)}^{o}=\frac{1}{4 \pi} \int_{0}^{\infty} \frac{d l_{1}}{l_{1}^{1 / 2}} \int_{0}^{\infty} \frac{d l_{2}}{l_{2}^{1 / 2}} e^{-\left(l_{1}+l_{2}\right)\left(\mu_{B}+w\right)} \frac{1}{l_{1}+l_{2}}$.

To regularize this divergent integral, we take a derivative with respect to $\tau_{n}$,

$$
\frac{\partial \mathcal{F}_{(1)}^{o}}{\partial \tau_{n}}=-\frac{1}{4} \frac{\partial w}{\partial \tau_{n}} \frac{1}{\mu_{B}+w} .
$$

Integrating over $\tau_{n}$ again gives

$$
\mathcal{F}_{(1)}^{o}=-\frac{1}{4} \log \left(\mu_{B}+w\right)+a,
$$

where $a$ is the integration constant independent of $\tau_{n}$. If the constant $a$ is fixed as $-\frac{1}{2} \log (\sqrt{2} i)$, then the result is 
consistent with (62) recalling (46). This demonstrates that the matrix calculation coincides with that of the open $\mathrm{KdV}$ results. We expect this holds for higher $\bar{g}$ solution.

\section{GENERATING FUNCTION OF INTERSECTION THEORY WITH BOUNDARIES}

GF of the OIT satisfies the open KdV hierarchy (27) and the open Virasoro constraints (24). So, one can evaluate this GF solving the open KdV hierarchy, or, equivalently, Virasoro constraints, using the initial conditions

$$
F^{o}\left(t_{0}, t_{i>0}=0, s\right)=\frac{1}{\lambda}\left(s t_{0}+\frac{s^{3}}{3 !}\right) .
$$

The solution is unique [36].

In this section, we will find the GF of OIT in a different way. Namely, we demonstrate that the Laplace transform (32) converts the GF of a particular topological solution of the BMG in $\mu_{B}$ space into the GF of OIT in $s$ space,

$$
e^{F^{o}(s)}=\int d \mu_{B} e^{-s \mu_{B} / \lambda} e^{F^{o}\left(\mu_{B}\right)} .
$$

If the GF obeys the open KdV hierarchy and the GF on a disk ( $\bar{g}=0$ contribution) satisfies the initial condition (68), then higher $\bar{g}>0$ contribution should also work due to the uniqueness of the solution.

\section{A. Generating function on a disk}

Note that the Laplace transform (69) reduces to the Legendre transform for $\bar{g}=0$ [5],

$$
F_{(0)}^{o}(t, s)=F_{(0)}^{o}\left(t, \mu_{B}\right)-s \mu_{B},
$$

and the boundary parameters, $s$ and $\mu_{B}$, are related through

$$
s=\frac{\partial F_{(0)}^{o}\left(t, \mu_{B}\right)}{\partial \mu_{B}} \quad \text { or } \quad \mu_{B}=-\frac{\partial F_{(0)}^{o}(t, s)}{\partial s} .
$$

To get the GF of OIT, we have to take a particular GF on the $\mu_{B}$ side, $F_{(0)}^{o}\left(t, \mu_{B}\right)$. We claim that it is given by the disk GF (30) where $\tau_{0}$ substituted by $t_{0}$, and $v$ is the solution of the polynomial string equation (18) as $p$ tends to infinity. So GF of OIT is represented by (47) but with replacing the string polynomial $\mathcal{P}(\tau, v)$ with $P(t, v)$ in (18), and accordingly the GF is denoted by $F$ rather than $\mathcal{F}$ in the rest of this paper,

$F_{(0)}^{o}\left(t, \mu_{B}\right)=-\frac{i}{\sqrt{2 \pi}} \int_{0}^{\infty} \frac{d l}{l^{3 / 2}} e^{-l \mu_{B}} \int_{w}^{\infty} d v P^{(1)}(t, v) e^{-l v}$.

Here $w$ corresponds to the solution of $P(t, v)=0$, regarding the terms with the parameter set $\left\{t_{k}\right\}$ is treated as a perturbation.
Let us check if this OIT solution coincides with the GF in $s$ space. We use the Legendre transform with the conjugate variable $s$,

$s=\frac{\partial F_{(0)}^{o}}{\partial \mu_{B}}=\frac{i}{\sqrt{2 \pi}} \int_{0}^{\infty} \frac{d l}{l^{1 / 2}} e^{-l \mu_{B}} \int_{w}^{\infty} d v P^{(1)}(t, v) e^{-l v}$.

To get some idea about how to find the explicit form of $F_{(0)}^{o}(t, s)$, we start with $p=1$ case and move on to $p=2,3$ and $p=4$, and then extract generic features. Of course, for any $p$, the case with $p-1$ can be obtained if one puts $t_{p-1}=0$.

(i) $p=1$ case: In this, case only $t_{0}$ is present, $P^{(1)}=-1$, and $w=t_{0}$. Thus, (72) has the simple form

$$
F_{(0)}^{o}\left(t_{0}, \mu_{B}\right)=\frac{i}{\sqrt{2 \pi}} \int_{0}^{\infty} \frac{d l}{l^{5 / 2}} e^{-l\left(\mu_{B}+w\right)} .
$$

Note that this integral depends only on the sum $\mu_{B}+w$. This integral is divergent as $l \rightarrow 0$ and needs regularization to be finite. We note the differentiation which makes the integral finite,

$$
\frac{\partial^{2} F_{(0)}^{o}}{\partial \mu_{B}^{2}}=\frac{i}{\sqrt{2 \pi}} \int_{0}^{\infty} \frac{d l}{l^{1 / 2}} e^{-l\left(\mu_{B}+t_{0}\right)}=\frac{i}{\sqrt{2}}\left(\mu_{B}+w\right)^{-1 / 2} .
$$

After integration over $\mu_{B}$ once, we have

$$
s=\frac{\partial F_{(0)}^{o}}{\partial \mu_{B}}=i \sqrt{2}\left(\mu_{B}+w\right)^{1 / 2}
$$

discarding $\mu_{B}$-independent term. Likewise, one more integration gives

$$
F_{(0)}^{o}\left(t_{0}, \mu_{B}\right)=i \frac{2 \sqrt{2}}{3}\left(\mu_{B}+w\right)^{3 / 2} .
$$

We provide another derivation from (38) in Appendix B.

After the Legendre transformation, we have GF of OIT,

$$
F_{(0)}^{o}\left(t_{0}, s\right)=s w+\frac{s^{3}}{3 !}=s t_{0}+\frac{s^{3}}{3 !},
$$

which is exactly the same as the initial condition (68). (ii) $p=2$ case: In the presence of two variables $t_{0}$ and $t_{1}$, one has $P^{(1)}(t, v)=t_{1}-1$, which we also denote by $-\xi_{1}$, and $w=t_{0} /\left(1-t_{1}\right)$. As in the $p=1$ case, we can evaluate (72) after regularization, 


$$
\begin{gathered}
s=\frac{\partial F_{(0)}^{o}}{\partial \mu_{B}}=i \sqrt{2} \xi_{1}\left(\mu_{B}+w\right)^{1 / 2}, \\
F_{(0)}^{o}\left(t, \mu_{B}\right)=i \frac{2 \sqrt{2}}{3} \xi_{1}\left(\mu_{B}+w\right)^{3 / 2} .
\end{gathered}
$$

The Legendre transform results in GF,

$$
\begin{aligned}
F_{(0)}^{o}\left(t_{0}, t_{1}, s\right) & =\frac{s t_{0}}{1-t_{1}}+\frac{s^{3}}{3 !\left(1-t_{1}\right)^{2}} \\
& =s w+\frac{s^{3}}{3 !\left(1-t_{1}\right)^{2}},
\end{aligned}
$$

which is in the agreement with the results of [6].

As the number of $\mathrm{KdV}$ parameters increases, the evaluation becomes not easy to carry out. To simplify it, we note that the open string equation (26) at $\bar{g}=0$ has the form of differential equation

$$
D F_{(0)}^{o}=s,
$$

where

$$
D \equiv \frac{\partial}{\partial t_{0}}-\sum_{n \geq 0} t_{n+1} \frac{\partial}{\partial t_{n}}
$$

The inhomogeneous solution to Eq. (82) is $s w$. This can be seen as follows. Since $w$ is the solution of the string polynomial equation $P(t, v)=0$, its derivative $\partial P(t, w(t)) / \partial t_{0}=0$ satisfies an identity

$$
\frac{\partial w}{\partial t_{0}}=\sum_{n \geq 0} t_{n+1} \frac{\partial w}{\partial t_{n}}+1 .
$$

Thus, $s w$ satisfies (82). In addition, there exist solutions of the corresponding homogeneous equation. A homogeneous solution $f$ can be put as a function of $s$ and a convenient set of parameters $\xi_{1}, \ldots, \xi_{p-1}$,

$\xi_{n}=-\left.\frac{d^{n} P(t, v)}{d v^{n}}\right|_{v=w} \equiv-P^{(n)}(w)$ for $n=1, \cdots p-1$.

One may easily show that $D \xi_{n}=0$. Therefore, we may put GF at $\bar{g}=0$ as the following form:

$$
F_{(0)}^{o}(t, s)=s w+f(s, \xi),
$$

with the homogeneous solution $f$. This structure of GF is already seen in $p=1$ and 2 cases.

The variables $\xi_{n}$ are well known in the matrix models $[9,10]$. These variables are extremely convenient for investigation of the GF and correlation functions for the resolvents for the closed case. As the GF of the BMG can be related to the correlation function of the local operators in MG $[15,18]$, it is not very surprising that they also show up in the theory with boundary.

(iii) $p=3$ case: For the case $p=3$, the solution of the polynomial string equation $w$ has the form

$$
\begin{aligned}
w & =\frac{1-t_{1}}{t_{2}}\left(1-\sqrt{1-\frac{2 t_{0} t_{2}}{\left(1-t_{1}\right)^{2}}}\right) \\
& =\frac{t_{0}}{1-t_{1}}\left(\frac{2}{1+\sqrt{1-\frac{2 t_{0} t_{2}}{\left(1-t_{1}\right)^{2}}}}\right) .
\end{aligned}
$$

One finds

$$
\begin{aligned}
s & =\frac{\partial F_{(0)}^{o}\left(t, \mu_{B}\right)}{\partial \mu_{B}} \\
& =\sqrt{2} i\left(\mu_{B}+w\right)^{1 / 2}\left(\xi_{1}-\frac{2}{3} \xi_{2}\left(\mu_{B}+w\right)\right),
\end{aligned}
$$

where (assuming $t_{1}<1$ )

$$
\begin{aligned}
& \xi_{1}=-P^{(1)}(t, w)=1-t_{1}-t_{2} w \\
&=\sqrt{\left(1-t_{1}\right)^{2}-2 t_{0} t_{2}}, \\
& \xi_{2}=-p^{(2)}(t, w)=-t_{2} .
\end{aligned}
$$

Integrating over $\mu_{B}$, one has

$$
\begin{aligned}
F_{(0)}^{o}\left(t, \mu_{B}\right)= & \sqrt{2} i\left(\mu_{B}+w\right)^{3 / 2} \\
& \times\left(\frac{2}{3} \xi_{1}-\frac{4}{5 ! !} \xi_{2}\left(\mu_{B}+w\right)\right) .
\end{aligned}
$$

Noting that $\partial \xi_{1} / \partial t_{0}=\xi_{2} / \xi_{1}$, one can check that $F_{(0)}^{o}$ satisfies the BCE equation (45).

To find GF in $s$ space, we put $\mu_{B}$ in powers of $s$ by solving (88)

$$
\begin{aligned}
& \mu_{B}=-w-\frac{s^{2}}{2 \xi_{1}^{2}} \sum_{n=0}^{\infty} a_{n} z_{1}^{n} ; \\
& a_{n}=\frac{(-1)^{n}}{n+1}\left(\begin{array}{c}
3 n+1 \\
n
\end{array}\right),
\end{aligned}
$$

with $z_{1}=s^{2} \xi_{2} /\left(3 \xi_{1}^{3}\right)$. Then, using the second relation in (71), one can easily find GF directly by integrating over $s$, 


$$
F_{(0)}^{o}(t, s)=s w+\frac{s^{3}}{2 \xi_{1}^{2}} \sum_{n=0}^{\infty} \frac{a_{n} z_{1}^{n}}{(2 n+3)},
$$

which has the expected structure of (86).

(iv) $p=4$ case: For the case $p=4$, we have

$$
\begin{gathered}
s=\frac{\partial F_{(0)}^{o}}{\partial \mu_{B}}=\sqrt{2} i\left(\mu_{B}+w\right)^{1 / 2} \\
\times\left(\xi_{1}-\frac{2}{3 ! !} \xi_{2}\left(\mu_{B}+w\right)+\frac{4}{5 ! !} \xi_{3}\left(\mu_{B}+w\right)^{2}\right) .
\end{gathered}
$$

We note that (94) is a polynomial equation of the form

$$
\frac{1}{h_{0}}=\left(1+z_{1} h_{0}+z_{2} h_{0}^{2}\right)^{2},
$$

where $h_{0}=-2 \xi_{1}^{2}\left(\mu_{B}+w\right) / s^{2}, z_{1}=s^{2} \xi_{2} /\left(3 \xi_{1}^{3}\right)$ and $z_{2}=s^{4} \xi_{3} /\left(15 \xi_{1}^{5}\right)$. One can find $h_{0}$ as a series in $z_{i}$ 's,

$$
\begin{aligned}
h_{0} & =\sum_{n, m=0}^{\infty} a_{n, m} z_{1}^{n} z_{2}^{m} ; \\
a_{n, m} & =2 \frac{(-1)^{n+m}(3 n+5 m+1) !}{n ! m !(2 n+4 m+2) !},
\end{aligned}
$$

where $a_{n, 0}=a_{n}$ in (92), so that the solution (97) reduces to the one in (92) when $z_{2} \rightarrow 0$. This shows that

$$
\mu_{B}=-w-\frac{s^{2}}{2 \xi_{1}^{2}} \sum_{n, m=0}^{\infty} a_{n, m} z_{1}^{n} z_{2}^{m}
$$

Using the second relation in (71), one integrates $\mu_{B}$ over $s$ to find GF of the form

$$
F_{(0)}^{o}(t, s)=s w+\frac{s^{3}}{2 \xi_{1}^{2}} \sum_{n, m=0}^{\infty} \frac{a_{n, m} z_{1}^{n} z_{2}^{m}}{(2 n+4 m+3)} .
$$

(v) Arbitrary $p$ : In general, (73) provides the relation between $\mu_{B}$ and $s$ as follows:

$$
s=\frac{\partial F_{(0)}^{o}}{\partial \mu_{B}}=\sqrt{2} i \sum_{n=0}^{p-2} \frac{(-2)^{n} \xi_{n+1}\left(\mu_{B}+w\right)^{n+1 / 2}}{(2 n+1) ! !} .
$$

We find $F_{(0)}^{o}\left(t, \mu_{B}\right)$ by integrating (100) over $\mu_{B}$,

$$
F_{(0)}^{o}\left(t, \mu_{B}\right)=-\sqrt{2} i \sum_{n=0}^{p-2} \frac{(-2)^{n+1} \xi_{n+1}\left(\mu_{B}+w\right)^{n+3 / 2}}{(2 n+3) ! !} \text {. }
$$

Similar to (96), we rewrite (100) in the following form to find $\mu_{B}$ in power series of $s$,

$$
\frac{1}{h_{0}}=\left(1+\sum_{n=1}^{p-2} z_{n} h_{0}^{n}\right)^{2}, \quad z_{i}=\frac{s^{2 i} \xi_{i+1}}{(2 i+1) ! ! \xi_{1}^{2 i+1}},
$$

where $h_{0}$ is the same in (96): $h_{0}=-2 \xi_{1}^{2}\left(\mu_{B}+w\right) / s^{2}$. Then $h_{0}$ is given in a power series of $z_{k}$ 's,

$$
h_{0}=\sum_{n_{k} \geq 0} a_{n_{1}, \ldots, n_{p-2}} z_{1}^{n_{1}} \cdots z_{p-2}^{n_{p-2}}
$$

where the coefficient $a_{n_{1}, \ldots, n_{p-2}}$ has the form

$$
a_{n_{1}, n_{2}, \ldots, n_{p-2}}=2 \frac{(-1)^{n_{1}+n_{2}+\cdots+n_{p-2}}\left(1+3 n_{1}+5 n_{2}+\cdots+(2 p-3) n_{p-2}\right) !}{n_{1} ! n_{2} ! \ldots n_{p-2} !\left(2+2 n_{1}+4 n_{2}+\cdots+2(p-2) n_{p-2}\right) !} .
$$

By noting

$$
\mu_{B}=-w-\frac{s^{2}}{2 \xi_{1}^{2}} \sum_{n_{k} \geq 0} a_{n_{1}, \ldots, n_{p-2}} z_{1}^{n_{1}} \cdots z_{p-2}^{n_{p-2}}
$$

one has GF in power series of $z_{k}$ 's by integrating over $s$ following the second relation in (71),

$$
F_{(0)}^{o}(t, s)=s w+\frac{s^{3}}{2 \xi_{1}^{2}} \sum_{n_{i}=0}^{\infty} \frac{a_{n_{1}, \ldots, n_{p-2}} z_{1}^{n_{1}} \cdots z_{p-2}^{n_{p-2}}}{\left(3+2 n_{1}+4 n_{2}+\cdots+2(p-2) n_{p-2}\right)} .
$$

To get the complete GF of OIT, one should tend $p$ to infinity. The result (106) with formally replacing $p \rightarrow \infty$ accommodates all the dependence on the KdV parameters, part of which is consistent with the theorem provided by Pandharipande et al. [6] 
which deals with a special case restricted by the limit $t_{0} \rightarrow 0$. In this limit, one has $w \rightarrow 0, \xi_{1} \rightarrow 1-t_{1}, \xi_{i} \rightarrow-t_{i}(i \geq 2)$ and GF has the following form:

$$
\begin{aligned}
\left.F_{(0)}^{o}(t, s)\right|_{t_{0}=0}= & \sum_{n_{i}=0}^{\infty} \frac{\left(1+3 n_{1}+5 n_{2}+\ldots\right) !}{n_{1} ! n_{2} ! \ldots\left(3+2 n_{1}+4 n_{2}+\ldots\right) !} \\
& \times\left(\frac{t_{2}}{3 ! !}\right)^{n_{1}}\left(\frac{t_{3}}{5 ! !}\right)^{n_{2}} \cdots \frac{s^{3+2 n_{1}+4 n_{2}+\ldots}}{\left(1-t_{1}\right)^{2+3 n_{1}+5 n_{2}+\ldots}} .
\end{aligned}
$$

This provides the correlation numbers

$$
\begin{gathered}
\left\langle O_{\alpha_{1}} \ldots O_{\alpha_{\ell}} \sigma^{k}\right\rangle_{0}^{o}=\left.\frac{\partial^{\ell+k} F_{(0)}^{o}}{\partial t_{\alpha_{1} \ldots} \partial t_{\alpha_{\ell}} \partial s^{k}}\right|_{(t, s)=0} \\
=\frac{\left(1+\Sigma_{i=1}^{\ell}\left(2 \alpha_{i}-1\right)\right) !}{\prod_{i=1}^{\ell}\left(2 \alpha_{i}-1\right) ! !}
\end{gathered}
$$

Here we note that the number $k$ of marked points on the boundary is specified by the set $\left\{\alpha_{i}\right\}: k=$ $3+\sum_{i=1}^{\ell} 2\left(\alpha_{i}-1\right)$, which is clearly seen in (107).

\section{B. Higher $\bar{g}$ expansion}

The universal formula for the GF on the cylinder $(\bar{g}=1)$ in the $\mu_{B}$ picture $F_{(1)}^{o}\left(t, \mu_{B}\right)$ was obtained in Sec. III B (67),

$$
F_{(1)}^{o}\left(\mu_{B}\right)=-\frac{1}{4} \log \left(\mu_{B}+w\right)+c_{1},
$$

with $c_{1}$ being a constant to be determined later. We expect that this relation as well as the disk GF (101) and higher $\bar{g}$ contributions can be also extracted from the $\lambda$ expansion of the wave function formula (38).

For the cylinder, the relation between the GF in $s$ and $\mu_{B}$ pictures is given by (37)

$$
F_{(1)}^{o}(s)=F_{(1)}^{o}\left(\mu_{B}\right)-\frac{1}{2} \log \left(\frac{\partial^{2} F_{(0)}^{o}\left(\mu_{B}\right)}{\partial \mu_{B}^{2}}\right),
$$

which, with the help of (101), reduces to

$$
\begin{aligned}
F_{(1)}^{o}(s)= & -\left.\frac{1}{2} \log \left(\xi_{1}+\sum_{n=1}^{p-2} \frac{(-2)^{n} \xi_{n+1}\left(\mu_{B}+w\right)^{n}}{(2 n-1) ! !}\right)\right|_{\mu_{B}=\mu_{B}(s)} \\
& +c
\end{aligned}
$$

where $c$ is a constant given by $c=c_{1}-(1 / 2) \log (i / \sqrt{2})$. This expression has the power series expansion in $z_{i}$ 's if one uses the expression for $\mu_{B}$ in (105).

To find the constant $c$, we consider the case with $t_{k}=0$ for $k>2$. The disk amplitude for this case is given by (81), so the cylinder GF (112) is
$F_{(1)}^{o}(t, s)=-\frac{1}{2} \log \left(\xi_{1}\right)+c=-\frac{1}{2} \log \left(1-t_{1}\right)+c$.

From this expression, we can conclude that $c=0$, and thus $c_{1}=(1 / 2) \log (i / \sqrt{2})$.

For $p=3 F_{(1)}^{o}(s)$ gives, up to logarithmic term, the power series expansion in $z_{1}$,

$$
\begin{aligned}
F_{(1)}^{o}(s) & =-\frac{1}{2} \log \left(\xi_{1}-2 \xi_{2}\left(\mu_{B}+w\right)\right) \\
& =-\frac{1}{2} \log \left(\xi_{1}\right)-\frac{3}{2} z_{1}+\frac{21}{2} z_{1}^{2}-24 z_{1}^{3} \\
& +\frac{981}{8} z_{1}^{4}-\frac{6663}{10} z_{1}^{5}+3765 z_{1}^{6}+\cdots
\end{aligned}
$$

Let us compare this result with a solution to the open string equation and the open $\mathrm{KdV}$ in $\bar{g}$ expansion in the $s$ picture. The open string equation with $\bar{g} \geq 1$ becomes a homogeneous differential equation

$$
D F_{(\bar{g})}^{o}=0,
$$

where $D$ is defined by (83). Therefore, GF with $\bar{g} \geq 1$ is represented in terms of $\xi_{i}$, the solutions of the homogeneous equation

$$
F_{(\bar{g})}^{o}=F_{(\bar{g})}^{o}\left(\xi_{i}, s\right) \text { for } \bar{g} \geq 1 .
$$

In addition, the $s$-flow equation (28) in $\bar{g}$ expansion has the form

$\frac{\partial F_{(\bar{g})}^{o}}{\partial s}=\sum_{\bar{g}_{1}+\bar{g}_{2}=\bar{g}} \frac{1}{2}\left(\frac{\partial F_{\left(\bar{g}_{1}\right)}^{o}}{\partial t_{0}} \frac{\partial F_{\left(\bar{g}_{2}\right)}^{o}}{\partial t_{0}}\right)+\frac{1}{2} \frac{\partial^{2} F_{(\bar{g}-1)}^{o}}{\partial t_{0}^{2}}+\frac{\partial^{2} F_{(\bar{g} / 2)}^{c}}{\partial t_{0}^{2}}$,

which can be used to restrict further the form of (117). Finally, it should satisfy open $\mathrm{KdV}$ equation: for $n \geq 1$,

$$
\begin{aligned}
\frac{2 n+1}{2} \frac{\partial F_{(\bar{g})}^{o}}{\partial t_{n}}= & \sum_{\bar{g}_{1}+\bar{g}_{2}=\bar{g}}\left(\frac{\partial F_{\left(\bar{g}_{1}\right)}^{o}}{\partial s} \frac{\partial F_{\left(\bar{g}_{2}\right)}^{o}}{\partial t_{n-1}}\right)+\frac{\partial^{2} F_{(\bar{g}-1)}^{o}}{\partial s \partial t_{n-1}} \\
& +\frac{1}{2} \sum_{\bar{g}_{1}+2 g_{2}=\bar{g}}\left(\frac{\partial F_{\left(\bar{g}_{1}\right)}^{o}}{\partial t_{0}} \frac{\partial^{2} F_{\left(g_{2}\right)}^{c}}{\partial t_{0} \partial t_{n-1}}\right) \\
& -\frac{1}{4} \frac{\partial^{3} F_{((\bar{g}-1) / 2)}^{c}}{\partial t_{0}^{2} \partial t_{n-1}} .
\end{aligned}
$$

It is to be noted that $F_{(\bar{g})}^{o}$ has an important parity property in $s$,

$$
F_{(\bar{g})}^{o}\left(\xi_{i},-s\right)=(-1)^{\bar{g}+1} F_{(\bar{g})}^{o}\left(\xi_{i}, s\right) .
$$

The parity property is already seen in (106) of Sec. IV B where $\mathrm{GF}_{(0)}^{o}(t, s)$ is odd in $s$. The general proof can be done 
using the dimension of the moduli space given in (21): $\bar{g}+k$ must always be odd [37]. Since $k$ denotes the power of $s$ in GF, one concludes (106). The parity property is also consistent with the SD considered in IT. Since $F^{o}$ is scale free as seen in (24), each term in $\bar{g}$ expansion is also scale free. Noting that $\mathrm{SD}$ of $\lambda$ is $3 / 2$, one has $\mathrm{SD}$ of $F_{(0)}^{o}$ is $3 / 2$, which is obvious in (106). (SD of $s$ and $w$ are $1 / 2$ and 1 , respectively, and SD of $\xi_{i}$ and $z_{i}$ are 0 .) Therefore, the $\bar{g}$ expansion of $F^{o}$ shows that $\mathrm{SD}$ of $F_{(\bar{g})}^{o}$ is $3(\bar{g}+1) / 2$. This shows that $\mathrm{SD}$ of GF with $\bar{g}$ even is a half-odd integer. Since SD of KdV parameter $t_{n}$ is an integer, the only way to have the half-odd integer SD is the quantity proportional to odd power of $s$ which is reflected in the parity (120).

Let us provide a few simple checks of the GF expression (112) by restricting the situation where most of the KdV parameters are turned off, though the expression (112) is valid for any integer $p$.

(i) $p=2$ case: At $\bar{g}=1$, the open string equation shows that $\partial F_{(1)}^{o} / \partial t_{0}=0$. Applying this condition to the $s$-flow equation, with the help of (81), one has $\partial F_{(1)}^{o} / \partial s=0$. This result is consistent with that $F_{(1)}^{o}$ is the even function of $s$. Based on this fact, the open $\mathrm{KdV}$ has the form

$$
\begin{aligned}
\frac{3}{2} \frac{\partial F_{(1)}^{o}}{\partial t_{1}} & =\frac{\partial^{2} F_{(0)}^{o}}{\partial s \partial t_{0}}-\frac{1}{4} \frac{\partial^{3} F_{(0)}^{c}}{\partial t_{0}^{3}} \\
& =\left(1-\frac{1}{4}\right) \frac{\partial w}{\partial t_{0}}=\frac{3}{4} \frac{1}{\left(1-t_{1}\right)} .
\end{aligned}
$$

The solution is given as

$$
F_{(1)}^{o}=-\frac{1}{2} \log \left(1-t_{1}\right),
$$

which coincides with (113).

(ii) $p=3$ case: In this case, noting that $F_{(1)}^{o}$ is scale free and an even function of $s$, we can find it in terms of scale-free parameters, $\xi_{1}$ and $z_{1}$. A direct evaluation shows that $F_{(1)}^{o}$ given by

$$
\begin{aligned}
F_{(1)}^{o}= & -\frac{1}{2} \log \left(\xi_{1}\right)+\sum_{n=1}^{\infty} \sum_{k=0}^{n-1} \frac{3(-1)^{n}}{2 n}\left(\begin{array}{c}
3 k \\
k
\end{array}\right) \\
& \times\left(\begin{array}{c}
3 n-3 k-2 \\
n-k-1
\end{array}\right) z_{1}^{n}
\end{aligned}
$$

solves the $s$-flow equation, coincides with (115), and its expansion in a power series in $t_{i}$ and $s$ gives

$$
\begin{aligned}
F_{(1)}^{o}= & \frac{t_{1}}{2}+\frac{t_{1}^{2}}{4}+\frac{s^{2} t_{2}}{2}+\frac{t_{0} t_{2}}{2}+\frac{3 s^{2} t_{1} t_{2}}{2} \\
& +t_{0} t_{1} t_{2}+3 s^{2} t_{1}^{2} t_{2}+\frac{3 t_{0} t_{1}^{2} t_{2}}{2}+\ldots,
\end{aligned}
$$

reproducing the result provided explicitly by [11] under an appropriate identification of the parameters.

\section{SUMMARY AND DISCUSSION}

We investigated the relation between the two-dimensional minimal gravity (Lee-Yang series) on Riemann surfaces with boundaries $\left(\mu_{B}\right.$, the boundary cosmological constant) and open intersection theory ( $s$, the source of the boundary marked point). The generating functions of both theories are considered as solutions to the open KdV hierarchy and string equation. Since there are many solutions to the open KdV hierarchy with different analytic properties, one needs a proper way to identify the right solution.

We use the conjecture that the generating function of the minimal gravity with $\mu_{B}$ and that of the intersection theory with $s$ is related by the Laplace transform. The generating function on a disk corresponds to the leading contribution to the Laplace transform, which reduces to the Legendre transform. We obtain the generating function of the intersection theory from that of the minimal gravity using the Legendre transform and confirm that the generating function of each theory belongs to a different solution sector of the open KdV hierarchy and string equation.

Based on this relation, we provide a systematic way to find the generating function in $\bar{g}$ expansion $(\bar{g}$ denotes the genus of the doubled Riemann surfaces, equivalent to the Euler characteristic). As the first nontrivial example of the machinery, we provide an explicit form of the generating function of open intersection theory on a cylinder $(\bar{g}=1)$, from that of the minimal gravity through the Laplace transform.

Higher $\bar{g}$ expansion is a more challenging problem. It will be interesting to find the generating function of the intersection theory through the Laplace transform and compare it with the combinatoric expression in $s$ space for the all-genera generating function [30]. It is to be noted that a given term of the $\bar{g}$ expansion contains contributions from the several topologically distinct surfaces. For example, $\bar{g}=2$ contains two different geometries: pants and kettle. For the open intersection theory, the contributions of different types of surfaces can be traced by the extension of the generating function $[37,38]$. However, the computations of the generating function of the minimal gravity with boundaries with topological structure different form the sphere with arbitrary number of boundaries is still not known, but can be extracted from the relation in terms of the closed GF $[32,33]$ or the matrix model computations $[15,18]$.

It is clear that the correlation functions in $\mu_{B}$ space present the nonanalytic behavior (square root branch), which is useful to describe the correlation numbers of primary operators. On the other hand, the correlation functions of the intersection theory in $s$ space show the polynomial behavior and are suited to describe the correlation numbers of the descendants. It is interesting to note that the very different role of the two theory spaces when related with the Laplace transform was used in [39] to solve 
the cosmological problem using the cosmological constant and its conjugate variable.

Another interesting problem is the description of the boundary gravitational descendants in open intersection theory introduced in $[31,36]$ and further investigated in $[11,37,38,40]$. This extended theory has a nice KontsevichPenner matrix model description. This identification immediately leads to the integrability of the deformed model, which is shown to be the tau function of the KP hierarchy. It would be interesting to find the minimal gravity counterpart of this deformed model. From the point of minimal gravity, this should correspond to consideration of the gravitating operators on boundaries and different types of the boundary conditions. The meaning of the Laplace transform from the point of view of the matrix integrals is not clear at the moment.

Finally, one may expect that the idea of this paper can be extended to the case of the so-called r-spin open intersection numbers [41]. One may relate this theory with $M(q, p)$ series of minimal gravity in terms of the $A_{q-1}$ Frobenius manifolds [23,24,42-44]. It would be interesting to apply the Laplace transform to investigation of the open $p-q$ duality. We are going to come back to these topics in the future publications.

\section{ACKNOWLEDGMENTS}

The work of H.M. and C.R. was partially supported by National Research Foundation of Korea Grant No. 2017R1A2A2A05001164, and of A. A. by Grant No. IBS-R003-D1 and by RFBR Grant No. 17-01-00585. A. A. would also like to thank Vasily Pestun for his hospitality at IHES (supported by the European Research Council under the European Union's Horizon 2020 research and innovation programme, QUASIFT Grant No. 677368), where this project was completed.

\section{APPENDIX A: OPEN KdV OF MINIMAL GRAVITY ON A DISK}

We provide a simple way to prove that GF (30) satisfies the open KdV hierarchy (31). According to (30),

$$
\frac{\partial \mathcal{F}_{(0)}^{o}}{\partial \tau_{n-1}}=-\frac{i}{\sqrt{2 \pi}} \int_{0}^{\infty} \frac{d l}{l^{1 / 2}} e^{-l \mu_{B}} \int_{\tau_{0}}^{\infty} d x \frac{\partial v}{\partial \tau_{n-1}} e^{-l v(x)},
$$

where we put $\frac{\partial v}{\partial \tau_{0}}=\frac{\partial v}{\partial x}$, and using the string equation (5), we have

$$
\frac{\partial \mathcal{F}_{(0)}^{o}}{\partial \tau_{n}}=-\frac{i}{\sqrt{2 \pi}} \int_{0}^{\infty} \frac{d l}{l^{1 / 2}} e^{-l \mu_{B}} \int_{w}^{\infty} d v \frac{v^{n}}{n !} e^{-l v},
$$

where we change the integration variable from $x$ to $v$. If one multiplies (A2) by $\mu_{B}$ (and uses $\tau_{n-1}$ instead of $\tau_{n}$ for later convenience), the result can be put in terms of the derivatives of $l$,
$-\mu_{B} \frac{\partial \mathcal{F}_{(0)}^{o}}{\partial \tau_{n-1}}=-\frac{i}{\sqrt{2 \pi}} \int_{0}^{\infty} \frac{d l}{l^{1 / 2}}\left(\frac{\partial e^{-l \mu_{B}}}{\partial l}\right) \int_{w}^{\infty} d v \frac{v^{n-1}}{(n-1) !} e^{-l v}$.

Using the integration by parts of $l$, one has

$$
\begin{aligned}
-\mu_{B} \frac{\partial \mathcal{F}_{(0)}^{o}}{\partial \tau_{n-1}}= & -\frac{i}{\sqrt{2 \pi}} \int_{0}^{\infty} \frac{d l}{l^{1 / 2}} e^{-l \mu_{B}}\left(v+\frac{1}{2 l}\right) \\
& \times \int_{w}^{\infty} d v \frac{v^{n-1}}{(n-1) !} e^{-l v}
\end{aligned}
$$

where the surface term vanishes. We may subtract $\frac{\partial \mathcal{F}_{(0)}^{o}}{\partial \tau_{n}}$ from the above,

$$
\begin{aligned}
-\mu_{B} & \frac{\partial \mathcal{F}_{(0)}^{o}}{\partial \tau_{n-1}}-\left(n+\frac{1}{2}\right) \frac{\partial \mathcal{F}_{(0)}^{o}}{\partial \tau_{n}} \\
= & -\frac{1}{2} \frac{i}{\sqrt{2 \pi}} \int_{0}^{\infty} \frac{d l}{l^{1 / 2}} e^{-l \mu_{B}} \frac{1}{l}\left(1-\frac{v l}{n}\right) \\
& \times \int_{w}^{\infty} d v \frac{v^{n-1}}{(n-1) !} e^{-1 v}
\end{aligned}
$$

and simplify the result

$$
\begin{aligned}
- & \frac{1}{2} \frac{i}{\sqrt{2 \pi}} \int_{0}^{\infty} \frac{d l}{l^{1 / 2}} e^{-l \mu_{B}} \frac{1}{l} \int_{w}^{\infty} d v \frac{d}{d v}\left[\frac{v^{n}}{n !} e^{-l v}\right] \\
= & \frac{w^{n}}{n !} \times \frac{1}{2} \frac{i}{\sqrt{2 \pi}} \int_{0}^{\infty} \frac{d l}{l^{1 / 2}} e^{-l \mu_{B}} \frac{1}{l} e^{-l w} .
\end{aligned}
$$

One may use the string equation (5) to get

$$
\frac{\partial^{2} \mathcal{F}_{(0)}^{c}}{\partial \tau_{0} \tau_{n-1}}=\frac{w^{n}}{n !}
$$

In addition, the rest term has the form

$$
\begin{aligned}
& \frac{i}{\sqrt{2 \pi}} \int_{0}^{\infty} \frac{d l}{l^{1 / 2}} e^{-l \mu_{B}} \frac{e^{-1 w}}{l} \\
& \quad=\frac{i}{\sqrt{2 \pi}} \int_{0}^{\infty} \frac{d l}{l^{1 / 2}} e^{-l \mu_{B}} \int_{w}^{\infty} d v e^{-l v}=-\frac{\partial \mathcal{F}_{(0)}^{o}}{\partial \tau_{0}} .
\end{aligned}
$$

Collecting all the results, we have the open KdV hierarchy (31)

$$
\frac{2 n+1}{2} \frac{\partial \mathcal{F}_{(0)}^{o}}{\partial \tau_{n}}=-\mu_{B} \frac{\partial \mathcal{F}_{(0)}^{o}}{\partial \tau_{n-1}}+\frac{1}{2} \frac{\partial \mathcal{F}_{(0)}^{o}}{\partial \tau_{0}} \frac{\partial^{2} \mathcal{F}_{(0)}^{c}}{\partial \tau_{0} \partial \tau_{n-1}}
$$




\section{APPENDIX B: ANOTHER DERIVATION OF GENERATING FUNCTION ON A DISK}

Here we demonstrate how (38) gives the GF of BMG on a disk for the simplest case (77), where all the $\mathrm{KdV}$ parameters are turned off $t_{i>0}=0$, except $t_{0}$. Extracting the leading term in a series expansion in $\lambda$ of the logarithm of (38), one finds

$F_{(0)}^{o}(t, z)=\sum_{k \geq 0}\left(t_{k}-\delta_{k, 1}\right) \frac{z^{2 k+1}}{(2 k+1) ! !}-\sum_{k} \frac{(2 k-1) ! !}{z^{2 k+1}} \frac{\partial F_{(0)}^{c}}{\partial t_{k}}$.

Recalling that

$F_{(0)}^{c}=\frac{1}{2} \int_{0}^{w} P^{2}(t, v) d v ; \quad P(t, v)=-v+\sum_{m=0}^{\infty} t_{m} \frac{v^{m}}{m !}$,

one has

$$
\frac{\partial F_{(0)}^{c}}{\partial t_{n}}=-\frac{w^{n+2}}{(n+2) n !}+\sum_{m=0}^{\infty} \frac{t_{m}}{m ! n !} \frac{w^{m+n+1}}{(m+n+1)},
$$

whose evaluation at $t_{i>0}=0$ results in

$\left.\frac{\partial F_{(0)}^{c}}{\partial t_{n}}\right|_{t_{i>0}=0}=-\frac{t_{0}^{n+2}}{(n+2) n !}+\frac{t_{0}^{n+2}}{(n+1) !}=\frac{t_{0}^{n+2}}{(n+2) !}$.

Making $t_{i>0}$ turn off, one obtains

$$
\begin{aligned}
F_{(0)}^{o}\left(t_{0}, z\right) & :=\left.F_{(0)}^{o}(t, z)\right|_{t_{i>0}=0} \\
& =t_{0} z-\frac{z^{3}}{3}-\sum_{k=0}^{\infty} \frac{(2 k-1) ! !}{z^{2 k+1}} \frac{t_{0}^{k+2}}{(k+2) !} .
\end{aligned}
$$

Substituting $z=i \sqrt{2 \mu_{B}}$, one immediately sees, noting $(-1) ! !=1$, that this is a series expansion of (77): $F_{(0)}^{o}\left(t_{0}, \mu_{B}\right)=i \frac{2 \sqrt{2}}{3 \lambda}\left(\mu_{B}+t_{0}\right)^{3 / 2}$ for large values of $\left|\mu_{B}\right|$.
[1] E. Witten, Two dimensional gravity and intersection theory on moduli space, Surv. Diff. Geom. 1, 243 (1991).

[2] M. Konstevich, Intersection theory on the moduli space of curves and the matrix Airy function, Commun. Math. Phys. 147, 1 (1992).

[3] A. Belavin, M. Bershtein, and G. Tarnopolsky, A remark on the three approaches to 2D quantum gravity, JETP Lett. 93, 47 (2011).

[4] A. Bawane, H. Muraki, and C. Rim, Open KdV hierarchy and minimal gravity on disk, Phys. Lett. B 783, 183 (2018).

[5] H. Muraki and C. Rim, Open KdV hierarchy of $2 d$ minimal gravity of Lee-Yang series, arXiv:1808.07304.

[6] R. Pandharipande, J. P. Solomon, and R. J. Tessler, Intersection theory on moduli of disks, open $\mathrm{KdV}$ and Virasoro, arXiv:1409.2191.

[7] M. Fukuma, H. Kawai, and R. Nakayama, Continuum Schwinger-Dyson equations and universal structures in two-dimensional quantum gravity, Int. J. Mod. Phys. A 06, 1385 (1991).

[8] R. Dijkgraaf, H. Verlinde, and E. Verlinde, Loop equations and Virasoro constraints in nonperturbative 2-D quantum gravity, Nucl. Phys. B348, 435 (1991).

[9] C. Itzykson and J. B. Zuber, Combinatorics of the modular group. 2. The Kontsevich integrals, Int. J. Mod. Phys. A 07, 5661 (1992).

[10] J. Ambjorn, L. Chekhov, C. F. Kristjansen, and Y. Makeenko, Matrix model calculations beyond the spherical limit, Nucl. Phys. B404, 127 (1993); Erratum, Nucl. Phys. B449, 681(E) (1995).
[11] A. Alexandrov, Open intersection numbers, KontsevichPenner model and cut-and-join operators, J. High Energy Phys. 08 (2015) 028.

[12] V. G. Knizhnik, A. M. Polyakov, and A. B. Zamolodchikov, Fractal structure of 2D quantum gravity, Mod. Phys. Lett. A 03, 819 (1988).

[13] V. A. Kazakov, I. K. Kostov, and A. A. Migdal, Critical properties of randomly triangulated planar random surfaces, Phys. Lett. 157B, 295 (1985).

[14] V. A. Kazakov, The appearance of matter fields from quantum fluctuations of 2D gravity, Mod. Phys. Lett. A 04, 2125 (1989).

[15] G. W. Moore, N. Seiberg, and M. Staudacher, From loops to states in 2-D quantum gravity, Nucl. Phys. B362, 665 (1991).

[16] A. A. Belavin and A. B. Zamolodchikov, On correlation numbers in 2D minimal gravity and matrix models, J. Phys. A 42, 304004 (2009).

[17] M. R. Douglas, Strings less than one dimension and generalized KdV hierarchies, Phys. Lett. B 238, 176 (1990).

[18] T. Banks, M. R. Douglas, N. Seiberg, and S. H. Shenker, Microscopic and macroscopic loops in nonperturbative twodimensional gravity, Phys. Lett. B 238, 279 (1990).

[19] D. Gross and A. Migdal, Nonperturbative Solution of the Ising Model on a Random Surface, Phys. Rev. Lett. 64, 717 (1990).

[20] P. H. Ginsparg, M. Goulian, M. R. Plesser, and J. ZinnJustin, (p, q) String actions, Nucl. Phys. B342, 539 (1990).

[21] A. Belavin, B. Dubrovin, and B. Mukhametzhanov, Minimal Liouville gravity correlation numbers from Douglas string equation, J. High Energy Phys. 01 (2014) 156. 
[22] B. Dubrovin, Integrable systems in topological field theory, Nucl. Phys. B379, 627 (1992).

[23] V. Belavin and Yu. Rud, Matrix model approach to minimal Liouville gravity revisited, J. Phys. A 48, 18FT01 (2015).

[24] K. Aleshkin, V. Belavin, and C. Rim, Minimal gravity and Frobenius manifolds: Bulk correlation on sphere and disk, J. High Energy Phys. 11 (2017) 169.

[25] A. Zamolodchikov, Perturbed conformal field theory on fluctuating sphere, arXiv:hep-th/0508044.

[26] E. Witten, On the Kontsevich model and other models of two-dimensional gravity, IASSNS-HEP-91-24.

[27] A. Marshakov, A. Mironov, and A. Morozov, On equivalence of topological and quantum 2-d gravity, Phys. Lett. B 274, 280 (1992).

[28] S. Kharchev, A. Marshakov, A. Mironov, A. Morozov, and A. Zabrodin, Towards unified theory of 2-d gravity, Nucl. Phys. B380, 181 (1992).

[29] A. Buryak and R. J. Tessler, Matrix models and a proof of the open analog of Witten's conjecture, Commun. Math. Phys. 353, 1299 (2017).

[30] R. J. Tessler, The combinatorial formula for open gravitational descendents, arXiv:1507.04951.

[31] A. Buryak, Equivalence of the open $\mathrm{KdV}$ and the open Virasoro equations for the moduli space of Riemann surfaces with boundary, Lett. Math. Phys. 105, 1427 (2015).

[32] C. V. Johnson, On integrable $c<1$ open string theory, Nucl. Phys. B414, 239 (1994).

[33] S. Dalley, C. V. Johnson, T. R. Morris, and A. Watterstam, Unitary matrix models and 2-D quantum gravity, Mod. Phys. Lett. A 07, 2753 (1992).
[34] R. Dijkgraaf and E. Witten, Developments in topological gravity, Int. J. Mod. Phys. A 33, 1830029 (2018).

[35] G. Ishiki and C. Rim, Boundary correlation numbers in one matrix model, Phys. Lett. B 694, 272 (2010).

[36] A. Buryak, Open intersection numbers and the wave function of the KdV hierarchy, Moscow Math. J. 16, 27 (2016).

[37] A. Alexandrov, A. Buryak, and R. J. Tessler, Refined open intersection numbers and the Kontsevich-Penner matrix model, J. High Energy Phys. 03 (2017) 123.

[38] A. Alexandrov, Open intersection numbers and free fields, Nucl. Phys. B922, 247 (2017).

[39] H. Kawai, Low energy effective action of quantum gravity and the naturalness problem, Int. J. Mod. Phys. A 28, 1340001 (2013).

[40] A. Alexandrov, Open intersection numbers, matrix models and MKP hierarchy, J. High Energy Phys. 03 (2015) 042.

[41] A. Buryak, E. Clader, and R. Tessler, Open r-spin theory and the Gelfand-Dickey wave function, J. Geom. Phys. 137, 132 (2019).

[42] M. Bertola and D. Yang, The partition function of the extended r-reduced Kadomtsev-Petviashvili hierarchy, J. Phys. A 48, 195205 (2015).

[43] A. Bawane, H. Muraki, and C. Rim, Dual Frobenius manifolds of minimal gravity on disk, J. High Energy Phys. 03 (2018) 134.

[44] K. Aleshkin and V. Belavin, Open minimal strings and open Gelfand-Dickey hierarchies, J. High Energy Phys. 02 (2019) 043. 